\title{
Dynamical quenching and annealing in self-organization multiagent models
}

\author{
E. Burgos ${ }^{1 *}$, Horacio Ceva ${ }^{1 \dagger}$, R.P.J. Perazzo ${ }^{2,3 \ddagger}$ \\ ${ }^{1}$ Departamento de Física, Comisión Nacional de Energía Atómica, \\ Avda. del Libertador 8250,1429 Buenos Aires, Argentina \\ ${ }^{2}$ Departamento de Física FCEN, Universidad de Buenos Aires, \\ Ciudad Universitaria - Pabellón 1, 1428 Buenos Aires, Argentina \\ ${ }^{3}$ Centro de Estudios Avanzados, Universidad de Buenos Aires, \\ Uriburu 950, 1114 Buenos Aires, Argentina
}

(21 December 2000)

\begin{abstract}
We study the dynamics of a generalized Minority Game (GMG) and of the Bar Attendance Model (BAM) in which a number of agents self-organize to match an attendance that is fixed externally as a control parameter. We compare the usual dynamics used for the Minority Game with one for the BAM that makes a better use of the available information. We study the asymptotic states reached in both frameworks. We show that states that can be assimilated to either thermodynamic equilibrium or quenched configurations can appear in both models, but with different settings. We discuss the relevance of the parameter $G$ that measures the value of the prize for winning in units of the fine for losing. We also provide an annealing protocol by which the quenched configurations of the GMG can progressively be modified to reach an asymptotic equlibrium state that coincides with the one obtained with the BAM.

PACS Numbers: 02.50.Le,05.40.-a, 64.60.Ak, 89.90.+n
\end{abstract}

\section{INTRODUCTION}

In recent times considerable attention has been given to the description of selforganization processes in multiagent models. These are geared to explain how (and which)

\footnotetext{
*e-mail burgos@cnea.gov.ar

†e-mail ceva@cnea.gov.ar

${ }^{\ddagger}$ perazzo@df.uba.ar
} 
individual decisions give rise to cooperative effects that show up in the behavior of the system as a whole. Such models have a wide number of interesting applications in a variety of situations that range from routing of messages in an information network to the way in which equilibrium is (dynamically) achieved by an ensemble of economic agents.

The main question posed in this line of analysis is how a system composed of multiple agents acting in a decentralized fashion is able to reach a state of "macroscopic order" in which individual decisions appear to be coordinated with one another. A well know example of this line of research is the Bar Attendance Model (BAM) proposed by B. Arthur in Ref [1]. This model has been developed to illustrate a case of coordination through a process that can be assimilated to inductive learning.

In the BAM a (large) number of individuals have to decide whether or not to go to a bar at a certain date. They share the common perception that attendance is not desirable when the number of people who are present exceeds a critical value, which is smaller than the total population of clients. Otherwise they derive utility from going to the bar.

A dynamics is proposed by which each agent individually updates her criteria to decide the attendance. These are based upon public information, that concerns the total attendance in the previous days. Agents use inductive reasoning to predict the number of customers in the following day.

It is simple to realize that the model is self-deceiving: if everybody decides to attend, crowding is produced and everybody loses. Everybody will then refrain to go the next day situation in which, again, everybody loses because many could go without crowding. In an iterated version of the model agents plan their attendance for the next few days. In this case it is more evident that the only stable solution is a situation in which a diversity of individual strategies compensate with each other saturating the capacity of the bar every day. The system self-organizes in a (dynamic) Nash equilibrium in which every agent optimizes their respective situation given what has been done by the rest of the players. In this case any player that changes unilaterally her rules ends up loosing. Such equilibrium is also Pareto optimal because no agent can improve her situation without harming that of some other player. If adaptation is stocastic, the attendance equilibrates at the accepted value with random fluctuations.

An alternative version of the BAM called the Minority Game (MG) has recently received much attention [2], [3], [4], [5]. The main feature of this framework is that agents derive utility whenever their choice coincides with that of the minority. Self deceiving is also evident within this framework. The MG has been presented as a simplified version of the BAM. In the present paper we concentrate in the approach of the MG introduced in ref. [5] and make a detailed comparison of the MG and the BAM. We will see that the MG, besides being a particular case of the BAM (namely one in which the optimal attendance has been fixed at half of the total number of customers) it makes a different use of the same information [6].

The treatments of the BAM have traditionally [1], [7] been based upon genetic algorithms [11]. Each agent is assumed to keep a set of strings that encode her possible attendance schedules for the following days. After that period each agent computes the outcome of all her possible plans and chooses the best to use it during the next period. The attendance schedules are considered as the "genomes" of a genetic algorithm and are updated by selection, random mutations and crossover. The BAM can be regarded as an iterated version of the MG, i.e. one in which players have to make their choices for several rounds of the same 
game. There is however one important difference. The BAM only involves (the iteration of) pure strategies where players either go or not go to the bar. The players in the MG have instead mixed strategies, i.e. they make their choice of going or not going with a given probability. The iterated pure strategies in the BAM undergo an adaptive process. Within the MG mixed strategies also change. Each agent randomly attends the bar (with a given probability) and collects points or pays fines depending that the attendance is below or above the acceptable level. The mixed strategy (i.e.her attendance probability) is changed when her account falls below a given threshold. Both the BAM and the probabilistic approach of ref. [5] can be regarded as mean field descriptions because only the aggregate effect of the whole ensemble of agents is relevant for both adaptive processes. On the other hand, the mixed strategy played by the agents in the MG can be assimilated to a time averaged version of the iterated attendace schedules of the players in the BAM.

In order to render possible a comparison between the MG and the BAM two elements have to be considered. On the one hand a generalized version of the MG (the GMG) has to be developed to describe situations traditionally considered within the BAM, namely cases in which the winning choice is to meet the one taken by an arbitrary fraction of the population, not just the minority. There are no systematic studies of this GMG. On the other hand a probabilistic (mixed strategy) mean field approach of the BAM has to be developed. There are a number of possibilities to define the mixed strategies for these two models. In the present paper we consider for the GMG an obvious extension of the adaptive updating dynamics that reproduces the well known selforganizing features of the MG. For the BAM we propose instead a mixed strategy that reproduces the results obtained with the usual treatments of this model that use iterated, pure strategies. It turns out that both dynamics are different. It happens that the one associated to the BAM makes a better use of the available information than in the GMG. Although in both frameworks - GMG and BAM - all agents have access to the same information, with the proposed BAM rules they are able to keep better track of the causes for winning or loosing thus making a more effective use of it than in the GMG. These seamingly minor changes produce widely different cooperative behaviors. The GMG leads in general to quenched states that are far from equilibrium. The BAM evolution leads instead to an asymptotic configuration that can be thought of as a thermodynamic equilibrium: it has lost memory of the initial conditions, the individual strategies continue to be updated at all times and yet the density distribution of attendance strategies remains stationary. In the following sections we discuss how both models yield similar results in a rather reduced portion of the space of parameters but turn out to be largely different in all the rest. We show that an essential parameter that governs the selforganized configuration of the system is determined by the rewards and punishments that are contained in the scoring of successes and failures of the agents. The control parameter is the value $G$ of the prize for winning relative to the fine for losing. Ordinarily the effect of this parameter has been disregarded and the only case studied in the literature is $G=1$. Quenching and equilibrium regions change significantly for $G \neq 1$.

In the next Section we describe and characterize the quenched state in the GMG and discuss the phase diagram that is found if $G$ is left as a free parameter. In Section 3 we compare this to the BAM. In Sect. 4 we provide an analytic discussion to support the results presented in the previous sections. In Sect. 5 we present an annealing protocol that makes use of the fact that quenching is a consequence of the memory stored in the system encoded 
by the points gained by the agents. The protocol allows to reach equilibrium using the MG dynamics by modifying progressively the quenched state removing the memory stored in the system.

\section{THE GMG MODEL}

We consider an $N$-agent system in which each has an mixed attendance strategy expressed by a probability $p_{i}(i=1,2, \ldots, N)$ to go to the bar. We take $n$ to be the acceptable level of attendance without crowding. The prescription to adapt the individual strategies is the following. If the attendance $A$ turns out to be $A \leq n$, and the agent chooses to go to the bar, she gains $G$ points. Otherwise, pays a fine of one point. The value $G$ is left as a free parameter .

The balance of gains and losses keeps a record of successes and failures of each agent. If the latter are more frequent than the former, her account ends up by falling below some fixed threshold (that we take to be 0). In this case her account is reset to zero and her strategy $p_{i}$ is updated. This is done by changing the value of $p_{i}$ by one chosen at random within the interval $\left(p_{i}-\delta p, p_{i}+\delta p\right)$. The witdth of this interval (we take $\delta p=.05$ ) is left unchanged during the whole process. Reflective boundary conditions for $p_{i}$ are used whenever necessary.

All $N$ agents have initially 0 points and their initial attendance strategies are chosen with some criterion. In the present section we restrict ourselves to consider the case in which all $p$ 's are initially chosen at random from the interval $[0,1]$ with uniform probability.

The state of the many agent system is well described by the density distribution $P(p)$ that gives the fraction of agents that have a strategy within the interval $(p, p+d p)$. In order to calculate this we generate an ensemble of $H$ histories of the $N$-agent system by evolving it during a fixed number of iterations and starting from statistically equivalent initial conditions. The density $P(p)$ is obtained by normalizing the frequency of occurrence of a given strategy in the whole set of the $H$ histories. The number of iterations is fixed by checking that a stationary configuration has been reached.

We also compute other parameters that describe the state of the system. One is the attendance $A(t)$ averaged over the $H$ histories as a function of the iteration number $t$; in the following we quote $M(t) \equiv A(t) / N$, to facilitate the comparison for different values of $N$. A second important parameter is the fraction of agents $C_{s}(t)$ that change their individual strategies as a function of the iteration number. This fraction provides a direct measure of the stability of the state that has been reached. We will use $P_{\infty}$ to refer to the asymptotic distribution, $t \rightarrow \infty$, whenever the system reaches an stationary state.

A well known result obtained within the framework of the pure MG is a strong polarization of the population. A distribution $P_{\infty}^{M G}(p)$ is reached in which essentially half of the agents always go to the bar while the other half never go . As a consequence $M(t) \rightarrow 1 / 2$ except for random fluctuations due to the updating of individual strategies that take place at all times [5], [9].

The evolution of a system with the GMG rules and $n \neq N / 2$ has been studied by Johnson [8] and by us [6]. If the reduced acceptable level of attendance $\mu \equiv n / N$ is close enough to $1 / 2$ an equilibrium situation is reached that resembles the MG results (see Fig.(1a)). Equilibrium is shown by the fact that $M \rightarrow \mu$ and $C_{s}(t)$ stabilizes at a constant, non zero value after a transient. In Fig. [1 $\mathrm{b}$ ) we show typical plots of $M(t)$ and $C_{s}(t)$ for this situation. 
On the contrary, if $\mu$ is larger than a critical value $\mu_{c r}$ (see below) a situation is reached in which no further updating of the strategies takes place ('quenched' configuration). In Fig. 2 we show an example of the distribution $P_{q}(p)$ that is obtained by setting $\mu>\mu_{c r}>1 / 2$.

The distribution $P_{q}(p)$ that is obtained strongly differs from $P_{\infty}^{G M G}(p)$. The fact that $C_{s}(t)$ tends exponentially to 0 (see Fig. $2 \mathrm{~b}$ )) indicates that a situation has been found that is completely different than the one commented before. This happens because the largest fraction of the population has gained many points and even though their accounts may fluctuate, they are not expected to fall below the threshold required to update their individual strategies. The consequence of this is that $M$ stabilizes at a value that may be far from $\mu$. While the situation for $\mu \sim 1 / 2$ can be thought as some kind of thermodynamic equilibrium, this case should instead be associated to a quenched configuration in which the state variables are rapidly fixed at values that are close to the initial conditions.

We have performed extensive numerical simulations to investigate this situation using values of $\mu$ above and below $1 / 2$ and several values of $G$. As expected an overall symmetry is found around $\mu=1 / 2$. This is because matching an attendance level greater than $1 / 2$ is equivalent as matching a symmetric absence level equally greater than $1 / 2$. As will shortly become evident symmetry is not strict. We discuss this point in Section 5 .

Several features that are displayed in the quenched distribution shown in Fig. 2 are worth to be remarked. In the first place it is found that $P_{q}(p)=0$ for $0<p<1 / 2$ and $\mu \gg 1 / 2$. This can easily be understood. When $\mu \gg 1 / 2$ the agents that have $p<1 / 2$ lose more frequently that they win and therefore update their attendance strategies more frequently increasing the value of $p$ whenever possible. Once their individual strategies are greater that $1 / 2$ the inverse happens, agents seldom lose points and therefore are not forced to update their individual strategies. This also justifies that the width of the hump near $p=1 / 2$ turns out to be only a function of $\delta p$.

The above arguments indicate that the shape of $P_{q}(p)$ strongly depends upon the initial conditions. This is the reason why we prefer to call this effect "quenching" rather that "freezing". The latter suggests a new ordering that bears no relationship with the initial conditions, while the former indicates that a dynamics has been imposed that suddenly reaches a fixed point leaving the internal parameters of the system at values that are close to the ones chosen with the initial conditions and that may be far from equilibrium.

In Fig. 3 we illustrate some cases in which quenching takes place. We plot the asymptotic value of the reduced attendance $M$ reached by the system as a function of $\mu$, displaying only the sector with $\mu>1 / 2$, for several values of $G$.

In Fig.(33 b) we also show a plot of the dispersion $\sigma_{M}$ of the values of $M$ obtained in all the $H$ histories of the ensemble $\left.\left(\sigma_{M}^{2}=\sum_{h}^{H}\left(A_{\infty}(h)-<A_{\infty}>_{H}\right)^{2}\right) / N H\right)$. This parameter plays the rôle of a susceptibility. It displays a peak located at the transition region and is essentially constant outside it. The finite width of the peak as well as the irregularities of $M$ in the transition region are to be attributed to the finiteness of the system.

It is possible to associate a critical value $\mu_{c r}$ with the change between both regimes. In the numerical experiments, the large fluctuations of $M$ near $\mu_{c r}$ are due to the fact that some of the histories that make up the ensemble happen to quench while others, purely due to random fluctuations, reach instead equilibrium. As $\mu$ increases, the proportion between both types of histories gradually changes leading to the situation in which all possible evolutions lead to quenched states. The peaked behavior of $\sigma_{M}$ at $\mu=\mu_{c r}$ supports the picture that a 
sort of critical phenomenon actually takes place in the system for a precise value of $\mu$.

There are several criteria to define $\mu_{c r}$. We have chosen to define it by the minimum value of $\mu$ for which the dynamics leads the system to a situation in which the whole population has accumulated a fixed gain, that we (arbitrarily) chose to be not less than 20 points. In

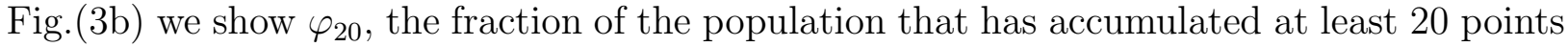
as a function of $\mu$. It is clear that if this happens any further changes in the distribution $P(p)$ or in the attendance are extremly unlikely to occur. Another possibility is to choose the value of $\mu$ that corresponds to the intersection of the lines that are associated to both regimes or the maximum of $\sigma_{M}$. Again the difference between these deffinitions are to be attributed to the finitness of the systems that we have studied.

The value of $\mu_{c r}$ depends both upon $G$ and $N$. Changes in $G$ have not been explored previously and cause important changes in the region in which quenching occurs as well as in the shape of the distribution $P_{\infty}^{G M G}(p)$. The effects of the value of $G$ in $\mu_{c r}$ can be seen in Fig. 3a). The general phase diagram (see Fig. 团) shows two regions that correspond to quenched and unquenched systems. The border between both regions is given by the function $\mu_{c r}(G)$. Finite size effects are also displayed in this figure through the values of $\mu_{c r}(G)$ for two values of $N$. The results displayed in Fig. 1 allows to conjecture that for an infinite system with $G=1$ and $\mu=1 / 2$ the usual settings of the Minority Game lead to an unstable quenched phase.

The changes in the distribution $P_{\infty}^{G M G}(p)$ that are due to changes in $\mu$ keeping $G$ fixed are shown in figs 1 and 2 and 5a). An increase in $\mu$ produces an increasingly asymmetric distribution. This hold up to the point in which $\mu>\mu_{\text {crit }}$ and quenching occurs giving rise to a humped distribution $P_{q}^{G M G}(p)$. The changes introduced by different values of $G$ are shown in Fig. 5b). The overall effect of this parameter is to increase the polarization, preserving the level of asymmetry fixed by $\mu$. Values of $G<1$ are reflected in distributions that are barely peaked at the corners. The polarization increases for $G=1$ and values of $G$ that are only slightly larger than 1 produce distributions $P_{\infty}^{G M G}(p)$ that vanish for all values of $p$ except in the close neighborhood of $p=0$ and $p=1$. Values of $G$ that are even larger places the system into the quenching region of the phase diagram because every successful initial move produces such a large gain that a revision of the strategy becomes extremely unlikely; therefore the evolution of the system produces only mild changes in the initial distribution.

\section{THE BAM MODEL}

The dynamics of the GMG does not keep track of the situations that lead to succeses or failures. This is instead stressed in the BAM. It has been customary to accept that agents derive utility in two situations: one when they decide to go to the bar and $M \leq \mu$ and also when they refrain to go to the bar and $M>\mu$.

We propose for the BAM an adaptive dynamics that is based upon the same information as in the GMG but uses it differently in order to keep track of the situations that lead to gains or losses i.e. by correlating them with the action of going or not going to the bar. To this end we introduce "points" and "credits". Points are gained or lost if the option of attending the bar is correct or not. Credits are instead gained or lost depending whether the option of not attending the bar is correct or not. We therefore carry a double account.

If an agent chooses to attend (not to attend) the bar and $M<\mu(M>\mu)$ gains a point (a 
credit). Otherwise, she loses a point (a credit). When either the number of points or the number of credits falls below some threshold, the corresponding account of points or credits is reset to zero and the strategy is changed in the same fashion as already mentioned for the GMG.

Note that the attendance strategy of an agent is changed by either of two reasons. Such updating rule makes use of information that is lost with the scoring rules used for the GMG. Suppose for instance that an agent having initially all her accounts in zero chooses to go to the bar one day in which $M<\mu$ and chooses not to go the next day when the attendance turns out to be again $M<\mu$. Within the GMG rules she ends up with zero points and her strategy is not updated. With the double account of the BAM she ends up instead with one positive point and one negative credit and is therefore forced to update her strategy [10].

We have performed numerical simulations using this dynamics with similar settings than those reported in the preceeding section. The results for the BAM are compared with those obtained with the GMG dynamics in Figs. 1.2. When quenching is absent in the GMG, the asymptotic distributions $P_{\infty}^{B A M}(p)$ can barely be distinguished from $P_{\infty}^{G M G}(p)$. For this case the values of the attendance $A(t)$ and $C_{s}(t)$ for the BAM are shown in Fig. 1 $\left.1 \mathrm{~b}\right)$. A strong difference however occurs when $\mu>\mu_{c r}$ and the GMG gets trapped in a quenched configuration. For the same settings the BAM converges instead to equilibrium. The asymptotic distribution $P_{\infty}^{B A M}$ shows a strong polarization and the attendance reaches the accepted level without difficulty. The nature of the asymptotic state has no difference with that for $\mu<\mu_{c r}$. This can be seen in Fig. 2b).

A more profound diference between the two model arises when the above analysis is repeated for $G \neq 1$. While the GMG dynamics can be checked to lead to quenched configurations for essentially all values of $G$, as shown in Fig. 4 , the BAM model displays no quenching independently of the value of $G$. Quenching can however occur in the BAM but for different initial conditions than a uniform distribution. We turn to this point in the next section.

\section{ANALYTIC DISCUSSION}

In this section we discuss analytically the relevant features of the density distribution $P(p)$ as well as the occurrence of quenching within both the GMG and the BAM.

\section{A. The density distribution}

The shape of the distribution $P(p)$ is the combined result of the actions of the whole ensemble of agents. Each player adjusts her attendance attempting to minimize her individual loss. When equilibrium is reached the resulting $P_{\infty}(p)$ for both GMG and BAM, concentrates the population in the immediate neighborhood of $p \simeq 0$ and $p \simeq 1$, plus an almost vanishing contribution from intermediate values. The ratio of the areas below the two peaks is, essentially, equal to $\mu /(1-\mu)$. An intuitive guess for $P(p)$ could well be instead a sharply peaked function centered at $p \simeq \mu$. The difference has to be sought in the properties of the distribution $\mathcal{P}(A)$ that gives the probability of occurrence of a party of $A$ customers attending the bar. The distribution $\mathcal{P}(A)$ is of course completely determined by 
$P(p)$. If $P(p) \simeq \delta(p-\mu)$ the corresponding $\mathcal{P}(A)$ turns out to be large for values of $A$ that differ from the optimal one, the highly polarized equilibrium distribution that is actually generated produces instead a $\mathcal{P}(A)$ that is much more concentrated near $A=N \mu$.

Let us assume without loss of generality that all the agents distribute themselves into $D$ different strategies $p_{i} ; i=1 \ldots D$ according to:

$$
P(p)=\sum_{i}^{D} \frac{n_{i}}{N} \delta\left(p-p_{i}\right)
$$

where $\sum_{i}^{D} n_{i}=N$. With this assumption, the distribution $\mathcal{P}(A)$ can be written as:

$$
\mathcal{P}(A)=\sum_{\ell_{1}=0}^{n_{1}} \ldots \sum_{\ell_{D}=0}^{n_{D}} \prod_{i}^{D}\left[\left(\begin{array}{c}
n_{i} \\
\ell_{i}
\end{array}\right) p_{i}^{\ell_{i}}\left(1-p_{i}\right)^{n_{i}-\ell_{i}}\right] \times \delta\left(A-\sum_{j}^{D} \ell_{j}\right) .
$$

The value $(A-N \mu)^{2}$ measures the departure from the optimal attendance, and therefore $\mathcal{C}^{2}=\sum_{A} \mathcal{P}(A)(A-N \mu)^{2}$ can be taken to measure the square of the average cost incurred by the ensemble of agents while adjusting their individual strategies [12].

Although Eq.2 is in general difficult to evaluate, $\mathcal{C}^{2}$ can be calculated without great difficulties. In fact if its definition is introduced into Eq.2, the summation over $A$ can be performed first, taking advantage of the $\delta\left(A-\sum_{j}^{D} \ell_{j}\right)$ and all the summations over the $\ell_{j}$ indices decouple from each other. It results

$$
\mathcal{C}^{2}=\sum_{\ell_{1}=0}^{n_{1}} \ldots \sum_{\ell_{D}=0}^{n_{D}} \prod_{i}^{D}\left[\left(\begin{array}{c}
n_{i} \\
\ell_{i}
\end{array}\right) p_{i}^{\ell_{i}}\left(1-p_{i}\right)^{n_{i}-\ell_{i}}\right] \times\left(N \mu-\sum_{j}^{D} \ell_{j}\right)^{2} .
$$

Moreover, from Eq.(11), $<p>=\sum_{i} n_{i} p_{i} / N$ and $<p^{2}>=\sum_{i} n_{i} p_{i}^{2} / N$, thus in the limit $D \rightarrow \infty$ one gets

$$
\mathcal{C}^{2}=N^{2}(\mu-<p>)^{2}+N\left(<p>-<p^{2}>\right)
$$

In Eq. $<p>$ stands for $\int p P(p) d p$. If $P(p)=1 / N$ as for the case of the initial conditions chosen in all the simulations in the preceeding sections, the dispersion turns out to be $\mathcal{C}^{2}=N^{2}(\mu-1 / 2)^{2}+N / 6$. We thus see that such initial condition is a good guess for the traditional settings of the $M G$ when $\mu \simeq 1 / 2$, but is indeed very poor for the GMG when $\mu \neq 1 / 2$.

The equilibrium result $\langle p\rangle=\mu$ that is always found, is seen to eliminate the $O\left(N^{2}\right)$ terms in $\mathcal{C}^{2}$. This is also achieved with the guess $P(p)=\delta(p-\mu)$. However, all $O(N)$ terms do not cancel because $\mathcal{C}^{2}=N \mu(1-\mu)$. If the two peaked equilibrium distribution is roughly approximated by $P(p)=\frac{n_{1}}{N} \delta\left(p-p_{1}\right)+\frac{n_{2}}{N} \delta\left(p-p_{2}\right)$ one readily sees that the $O\left(N^{2}\right)$ terms are eliminated when $n_{1} p_{1}+n_{2} p_{2}=\mu N$. The $O(N)$ terms also cancel if the two peaks are $p_{1}=0 ; n_{1}=N(1-\mu)$ and $p_{2}=1 ; n_{2}=N \mu$.

From this point of view, quenched states are far from optimizing the average aggregate $\operatorname{cost} \mathcal{C}^{2}$ because the corresponding $P_{q}(p)$ do not even cancel the $O\left(N^{2}\right)$ terms. The relaxation dynamics that tends to minimize individual losses is thus seen to also optimize a global parameter $\left(\mathcal{C}^{2}\right)$ only if equilibrium is reached. Otherwise the relaxation stops when no agent loses. This situation, in general, does not corresponds to a minimal average cost. 


\section{B. BAM}

In this section we discuss the occurrence of equilibrium and quenching within the BAM. This can only be made without any approximation if one assumes a distribution $P(p)=$ $\delta\left(p-p_{o}\right)$. This is seen to be appropriate except for $O(N)$ terms in the dispersion of party sizes.

Given an acceptable level of attendance $n$, if all agents have the same attendance strategy $p_{o}$, the probability of occurrence of a party of less than $n$ agents is $S\left(N, n, p_{o}\right)$ where

$$
S\left(N, n, p_{o}\right)=\sum_{i=0}^{n-1}\left(\begin{array}{c}
N-1 \\
i
\end{array}\right) p_{o}^{i}\left(1-p_{o}\right)^{N-1-i} .
$$

This is a continuous and monotonous decreasing function of $p_{o}$ and fulfills the following three conditions:

$$
\begin{aligned}
S(N, n, 0) & =1 \quad \forall n \\
S(N, n, 1) & =0 \quad \forall n \\
S\left(N, n+m, p_{o}\right) & \geq S\left(N, n, p_{o}\right) \quad \forall p_{o}, m>0
\end{aligned}
$$

If an agent decides to go to the bar, her account in points will remain equilibrated when the expected rewards equals the expected fine, i.e. when the attendance strategy $p_{p}$ that is common to all the agents fulfills:

$$
S\left(N, n, p_{p}\right)=\frac{1}{1+G}
$$

If an agent decides instead not to go to the bar a similar condition can also be written for her account in credits. This is:

$$
S\left(N, n+1, p_{c}\right)=\frac{G}{1+G}
$$

Given $G, N$ and $n$, the two equations (9) and (10) define the two roots $p_{p}$ and $p_{c}$ that are respectively the probabilities with which all agents have to go if they hope to equilibrate, respectively their accounts of points and credits.

Assume now that $G=1$. Bearing in mind Eq. ( 8) the two roots fulfill $p_{p}<p_{c}$. If the agents choose to attend the bar with a probability $p_{o}<p_{p}$, they gain points. On the other hand, if they choose to attend with probability $p_{o}>p_{c}$ they gain credits. However, due to the fact that $p_{p}<p_{c}$ if they gain points they lose credits and viceversa. In either case all agents will continue to update their strategies, quenching is not possible and equilibrium is eventually reached.

For a $\delta$-type distribution of strategies the only chance to have a quenched phase using the BAM dynamics is to find a situation in which $p_{c} \leq p_{p}$. For finite $N$ this can only happen if $G>1$ (see Fig. 6). From the above two equations it is easy to see that the root $p_{c}$ becomes smaller for increasing values of $G$ and the opposite happens for $p_{p}$. A value $G_{c r i t}^{B A M}$ can eventually be found in which both roots fulfill $p_{p}=p_{c} \equiv p_{\text {crit }}$. For any $G>G_{\text {crit }}^{B A M}$ the regions for winning points and credits start to overlap and quenching occurs within a narrow 
overlapping interval $\left(p_{c}, p_{p}\right)$. In Fig. 7 we show the two roots $p_{p}$ and $p_{c}$ as a function of $G$. It further turns out that for finite values of $N$ the value of $G_{c r i t}^{B A}$ is nearly independent of $\mu$.

The evolution of a $\delta$-type distribution centered at a value of $p_{o}$ depends upon the choice

of $G$ and $\mu$. If $G>G_{c r i t}^{B A M}$, and $p_{o}$ is inside the quenching interval $\left(p_{c}, p_{p}\right)$ the distribution will not change and the attendance $M$ will remain fixed at the value chosen for the initial conditions. This is so because because all agents win both points and credits. If $p_{o}$ lies outside of the quenching region, agents are forced to update their strategies either because they lose point or because they lose credits. The average attendance $M$ will approach the value $\mu$. Note that the average attendance and the average of individual strategies are completely equivalent i.e. $M=\int p P(p) d p$. Therefore when the average attendance approaches the quenching interval $\left(p_{c}, p_{p}\right)$, the evolution will progressively come to a stop. For values of $G$ up to $G \sim 5 G_{\text {crit }}$ the quenching interval $\left(p_{c}, p_{p}\right)$ is always small $\left(\left|p_{p}-p_{c}\right| \sim\right.$ .1) and the convergence to a quenched phase is in general very slow. As the mean of the distribution approaches the quenching interval, a smaller fraction of agents have to update their strategies and therefore the fraction $C_{s}(t)$ tends slowly to zero approaching very nearly a power law, for a considerable number $\left(\sim 10^{6}\right)$ of iterations. Since $G_{\text {crit }}^{B A M}$ is almost independent of $\mu$ this behaviour does not change with its particular value. In the cases in which $p_{o} \notin\left(p_{c}, p_{p}\right)$ the corresponding (asymmetric) distributions $P_{q}^{B A M}(p)$ associated to quenched configurations look similar to those associated to equilibrium because involve agents with all possible attendance strategies.

\section{GMG}

A similar analysis as the one performed for the BAM can be made for the GMG. If the system is prepared with a distribution $P_{0}(p)=\delta\left(p-p_{o}\right)$ the single account of points remains equilibrated provided that the probability of winning $P_{w}(N, n, p)$ fulfills:

$$
P_{w}\left(N, n, p_{o}\right)=p_{o} S\left(N, n, p_{o}\right)+\left(1-p_{o}\right)\left(1-S\left(N, n+1, p_{o}\right)\right)=\frac{1}{1+G}
$$

In Fig. 6 we show an example of the functions $S(N, n, p), S(N, n+1, p)$ and $P_{w}(N, n, p)$ for typical values of $N$ and $n$, and $n>N / 2$.

The situation for the GMG is completely different than for the BAM. The function $P_{w}(N, n, p)$ has one maximum in the interval $0 \leq p \leq 1$, and to a high degree of accuracy displays the symmetry $P_{w}(N, n, p)=P_{w}(N, N-n, 1-p)$. Symmetry however is not strict. To see this note that if $n=N$, going to the bar is always a winning option no matter the option of the rest of the agents. If $n=0$, the action of not going is not enough to win, because that depends upon the choice made by all the other agents.

A critical value $G_{c r}^{G M G}$ and a critical probability of attendance $p_{c r i t}^{G M G}$ can be defined for the GMG as the $p$ value that corresponds to the maximum of $P_{w}(N, n, p)$ given by Eq.( 11) through

$$
\begin{aligned}
P_{w}\left(p_{c r i t}^{G M G}\right) & =P_{w}^{\max }=\max _{p} P_{w}(N, n, p) \\
G_{c r}^{G M G} & =\frac{1}{P_{w}^{\max }}-1
\end{aligned}
$$


For a given value of $n$ and $N$ quenching is produced if $p=p_{c r}$ and $G \geq G_{c r}$. the values of $G_{c r}$ for the GMG are completely different from those of the BAM. While in the latter there exists a common value of $G_{c r}$ independent of $\mu$ this is not the case for the GMG (see Fig. (1)).

Above $G_{c r}$ and initial condition $P_{0}(p)=\delta\left(p-p_{1}\right)$, the quenching condition is fulfilled for any $p_{1}$ in the interval $p_{<}<p<p_{>}$that are the two roots of Eq.(11). Any system prepared with $p_{1}$ within that region will rapidly be quenched. Assume for the sake of concretness that we choose $\mu>0.5$. The system evolves quite differently if $p_{o}<p_{<}$or $p_{o}>p_{>}$. In the first case the whole population of agents is forced to update their strategies in order to change the individual $p$ 's in order to surpass the value $p_{<}$. Once this happens, updating progressively comes to a stops as the average attendance gets into the interval $\left(p_{<}, p_{>}\right)$and the system gets quenched with a value of $M$ below the accepted fraction $\mu$. The fact that $P_{w}(N, n, p)$ is such that $p_{>}$is very nearly similar to $\mu$ causes that for $p_{1}>p_{>}$the initial condition of a $\delta$-type distribution changes into $P_{\infty}^{G M G}(p)$ with a mean value that is very close to $\mu$ that can not be distinguished from an equilibrium distribution. In addition, updating of strategies continues to take place continuously. Examples are shown in Fig. 8 .

\section{Finite-size effects}

An estimate of the finite size effects of the models on the values of $G_{c r}$ and $p_{c r}$ can easily be made for the usual settings of the MG, by assuming a $\delta$-type distribution as an initial condition. For $\mu=1 / 2$, and from Eqs.(11) and (13) it follows immediately that $p_{\text {crit }}=1 / 2 \forall N$ and the value of $G_{\text {crit }}^{G M G}$ can directly be estimated from the value of $P_{w}^{G M G}(p=1 / 2)$ using Eq. (11) and standard asymptotic expansions. It turns out:i

$$
G_{c r}^{G M G}=\frac{\sqrt{ }[2 \pi N]+2}{\sqrt{ }[2 \pi N]-2}
$$

If this result remains valid for a uniform initial distribution, the usual settings for the MG $(G=1$ and $\mu=1 / 2)$ correspond to a critically quenched system.

In Fig 9a) are shown $G_{\text {crit }}^{G M G}(\mu=1 / 2)$ and $G_{\text {crit }}^{B A M}(\mu=1 / 2)$ as a function of $1 / N$. It is seen that $G_{\text {crit }}^{G M G} \simeq G_{\text {crit }}^{B A M}$ as $N \rightarrow \infty$. The corresponding values for $\mu \neq 1 / 2$ can be related to those shown in Fig. 9a). In the first place one should notice that the value of $G_{\text {crit }}^{B A M}$ is independent of $\mu$. On the other hand, the curves of $G_{c r i t}^{G M G}(\mu \neq 1 / 2)$ can be reduced to the one shown through $G_{c r i t}^{G M G}(\mu)=(a \mu+b) G_{c r i t}^{G M G}(\mu=1 / 2)$ to a very good degree of approximation ( $a$ and $b \sim$ constants, up to terms $O(1 / N)$ ).

In what refers to the values of $p_{\text {crit }}$ one can check (Fig. 9 $\left.\mathrm{b}\right)$ ) that $p_{\text {crit }}^{B A M}$ and $p_{\text {crit }}^{G M G}$ approach each other and tend to the common limiting value $p_{c r i t}^{\infty}=\mu$, as expected from a naïve estimate of the probability of attendance. One can also conjecture that $G_{c r i t}^{B A M}(\infty)=1$.

\section{ANNEALING}

The situations described hitherto indicate that outside a very restricted region of $\mu$ and for different initial conditions, the dynamics of the GMG rapidly approaches quenching. The many agent system stops evolving, the attendance remains fixed at a value that is far from the tolerated fraction $\mu$ and all the other internal parameters remain close to the initial 
conditions. Opposed to this, the BAM approaches always a distribution $P_{\infty}^{B A M}(p)$ that corresponds to what one expects of thermodynamics equilibrium: the attendance fluctuates around the expected fraction $\mu$, agents continue to update their individual strategies and $P_{\infty}^{B A M}(p)$ remains stationary.

There is a way to regain equilibrium within the framework of the GMG through an iterative procedure that can be regarded as an annealing protocol. The key point is to realize that quenching is produced by the memory stored in the points gained by the agents. In fact the quenched state remains unchanged because on the average agents continue to gain points. To correct the quenched phase, a procedure can be implemented that consists in obliterating periodically the memory of the system. This is donne removing all the points gained by all the agents in each iteration. In each annealing episode the system is allowed to evolve freely during a maximum of $n_{s}$ steps; at the end, several parameters are calculated: the fraction $\varphi_{5}$ of agents that have gained not less that 5 points, the relative attendance $M$, the fraction of histories $f_{h}$ where the system does not reach a quenched configuration in the allowed $n_{s}$ steps, and the fraction $f_{q}=n_{q} / n_{s}$, where $n_{q}$ is the actual number of evolution steps that are needed to quench the system.

The results obtained are shown in Fig.10 for two values of $G$ and $\mu=850 / 1001$. At the end of the first episode the distribution $P_{q}$ (Fig.10a)) that is found is entirely similar to the humped distribution shown in Fig2, and $\varphi_{5}=1$, thus corresponding to a quenched phase. As agents are repeatedly deprived of points some of them are increasingly forced to update their strategies. For $G=1$ (Fig.10b)) and during the first 30 annealing episodes very few steps are required in order to have $\varphi_{5}=1$; after $\sim 35$ annealing episodes a clear transition takes place that can be interpreted as a gradual melting of the system: the fraction $\varphi_{5}$ drops while $f_{h}$ and $f_{q}$ grow abruptly, almost reaching their maximum possible values. For $G=1.1$ (Fig.10c) ) this transition is not present as can be seen from the fact that $\varphi_{5}$ is constantly equal to one, while $f_{h}=0$. It is most remarkable, however, that the relative attendance $M$ still reaches the value $\mu$, as for $G=1$. In other words, the annealing protocol is seen to be able to drive the system towards $M \simeq \mu$ with or without a change of the phase of the system.

\section{CONCLUSIONS}

In the present work we have discussed the self organization properties of two multi agent models, the BAM and the GMG. We have shown the relevance of two control parameters. One is the fraction of the population of agents $\mu$ that has to be joined in order to make the correct decision. The other is $G$ that represents the gain produced by a correct decision, measured in units of the fine payed for making the wrong one. For the sake of concreteness we have borrowed the framework of the BAM in which the decision that has to be made is to go or not to a bar. The decision is the correct one if the fraction of the population that has decided to go does not exceed $\mu$.

The system under consideration lacks any interaction or internal correlation among the agents. Each agent feels the presence of the rest only through the value of an aggregate parameter that is the total attendance and rewards or penalties of individual actions are decided with respect to its particular value in each moment. Otherwise, agents act independently from each other. With these rules, the many agent system adapts and always reaches 
stable asymptotic configurations. These are, however, of quite different nature depending upon the values of the control parameters, of the initial conditions and on the information that is used to guide the individual actions.

The usual framework of the minority game, if generalized to take into account arbitrary values of $\mu$, gives rise either to states of equilibrium or to quenched configurations. The former have a density distribution of individual strategies that only depends upon the value of $G$ and $\mu$ and remains stationary while a fraction of the population $C_{s}$ continuously updates their strategies. Quenched states have instead frequency distributions that may be very different depending upon the initial conditions, while $C_{s}$ drops exponentially to zero in all cases.

A phase diagram for the GMG can be established in the $\mu-G$ plane. Quenching occurs outside a region limited by a critical line $\mu_{c r}(G)$. This border line has been obtained numerically for uniform initial conditions. If a $\delta$-type distribution is assumed analytic expressions can be obtained. In both cases finite size effects are found. A rigorous estimate can be made for an infinite system in this later case. It is found that if $N \rightarrow \infty$ the point $(G=1, \mu=1 / 2)$ belongs to the critical line. A numerical estimate of finite size effects for a uniform initial distribution is also in agreement with this result. It is interesting to note that these are the usual settings for the minority game widely used in the literature.

The shape of the distribution $P(p)$ is the combined result of the actions of many "independent' agents. In spite of such independence we have proven that the equilibrium distribution optimizes the statistical occurrence of the most convenient coordinated state or, equivalently, minimizes the average aggregate cost of the ensemble of agents.

A quenched state is reached when the individual updating of strategies stops. This should however not be considered to correspond to a "frozen" configuration. If this where the case, an internal ordered state would be found bearing no relationship with the initial conditions. Contrary to this, the quenched state is far from being unique, the "glassy" structure of the quenched phase lacks any preferred internal ordering. This should be regarded as the signature of the absence of interactions among the agents and has to be attributed purely to dynamic effects. While equilibrium is associated to the optimization of the global magnitude, this is no longer true for a quenched configuration.

Reaching a quenched phase is a direct consequence of the evolution dynamics contained in the rules for updating the individual strategies. This effect is evident when the GMG is compared to the dynamics arising from the BAM with mixed strategies, as we have presented here. Contrary to the GMG, when starting from a uniform distribution the BAM always reaches a state of equilibrium, independently of the values of $\mu$ and $G$. The two models differ in the use of the available information. While the GMG dynamics only keeps record of good and wrong decisions, the seemingly minor modifications implied in the BAM rules, allows to keep also track of the reasons for such successes and failures. The system thus keeps a better "memory" of the previous steps in the dynamics. The consequence of this is that the system always self organizes in such a way that $M \rightarrow \mu$.

The many agent system that has been presented here has several features that are reminiscent of the physics of many body systems. In the first place one should note that a quenched phase can be induced for critical values of $\mu$ and $G$. A sort of order parameter can be established with the relative attendance $M(t)$. Two regions can be individualized in parameter space, one in which $M(t) \rightarrow \mu$ and the other in which $M(t) \rightarrow M_{\infty} \neq \mu$. For 
critical values of $\mu$ and $G$, the system undergoes an internal transformation reflected by a peak in the dispersion $\sigma_{A}$ that reminds the behavior of the susceptibility at a second order phase transition.

Although the model does not contain any two body interaction, within this analogy "particles" accomodate themselves following an ocupational constraint (the maximum allowed attendance) very much in the same fashion as in many fermion system. To support this viewpoint, one could note that very few "particles" participate in the dynamics. The parameter $\mu$ fixes the total attendance and therefore plays the role of the chemical potential through an effective collective interaction. From this point of view individual gains should be related to (the negative of) a kind of single particle energy: those agents that have accumulated large gains are less likely to participate in the dynamics.

A resemblance can also be established between quenching in GMG and similar situations found in many body systems. In fact we have described a gradual modification of the quenched configuration through a procedure that is entirely similar to an annealing protocol. It turns out that a quenched state can gradually be "melted" into a state of equilibrium if all the points that have been gained by the agents are periodically eliminated. The points accumulated by the agents keep memory of the previous results and each remotion can be thought as an exchange of 'energy' with a thermal bath in order to reach equilibrium.

The concept of temperature has been introduced in the MG in several contexts. In all cases it is assimilated to random fluctuations. In this work we have not attempted to introduce the temperature. It is worthwhile to mention, however, that in fact there is an individual source of fluctuations present, associated with the update of $p_{i}$ described in Section II. We recall that every time a new value of $p_{i}$ has to be chosen, it is randomly selected from an interval of width $\delta p$ around it. Hence, this fluctuation acts as a source of fixed temperature, $T_{0}$ say, and can be recognized as the final reason that explains why the distribution function $P(p)$ does not simply converges to the sum of several $\delta$ 's. Hence, our work can be considered to describe the relaxation to equilibrium at that fixed temperature $T=0$, except when the annealing protocol is applied. This framework could be extended to a situation at $T \neq 0$ by introducing fluctuations allowing for instance individual agents to change probabilistically their assistance strategies.

The BAM has been worked out to show how internal coordination of markets or economic systems can derive from uncoordinated individual actions that are inspired by a common belief. It is a clear example in which an ensemble of agents with limited information about somebody else's decisions can nevertheless give rise to a self organized pattern within a scheme referred to as "bounded rationality". The relaxation process reminds what in economics is known as "tatonnement". Opposed to this, the "rational expectations" solution to the BAM or the GMG would be a distribution $P(p)=\delta(p-\mu)$ because it describes the only common action that is consistent with the general knowledge of all agents about everybody else's decisions concerning the acceptable attendance. It is interesting to note that this solution is in general far from equilibrium and hence is neither stationary nor optimal.

The Minority Game, regarded as a simplified version of the BAM has also been repeatedly presented as a schematic framework with similar interest for economics. However the occurrence of quenched asymptotic configurations in a straightforward generalization of the MG puts some limitations to the aplicability of the model to stylized economic situations. Quenched configurations are trivial examples of equilibria because the adaptation dynamic 
has stopped, but they are neither Nash equilibria nor Pareto optimal. In fact, if $M \neq \mu$ many individual situations can be improved without harming the situation of any other agent. It is intersting to note that such configurations are produced by the limited use that agents make of the available information. GMG can therefore safely be used if quenching is avoided or special situations are considered in which this is not particularly relevant. Otherwise, one should bear in mind that to simulate market environments it is mandatory to assume that the agents must make full use of all the available information.

E.B. has been partially supported by CONICET of Argentina, PICT-PMT0051;H.C. and R.P. were partially supported by EC grant ARG/b7-3011/94/27, Contract 931005 AR. 


\section{REFERENCES}

[1] Amer. Econ. Assoc. Papers Proc. 84, 406 (1994)

[2] D. Challet, Y.C. Zhang, Physica A246, 407 (1997); Physica A256, 514 (1998)

[3] R. Savit, R. Manuca, R. Riolo, Phys. Rev. Lett. 82, 2203 (1999)

[4] A. Cavagna, Phys. Rev. E59, R3783 (1999)

[5] N.F. Johnson, P.M. Hui, R. Jonson, T.S. Lo, Phys. Rev. Lett. 82, 3360 (1999)

[6] E. Burgos, H. Ceva, R. Perazzo, cond-mat /0007010

[7] D. Heymann, R.P.J. Perazzo and A. Schuschny, preprint

[8] N.F. Johnson, D.J.T. Leonard, P.M. Hui, T.S. Lo, cond-mat /9905939 v2. See also: N.F. Johnson, P.M. Hui, Dafang Zeng, C.W. Tai, Physica A269, 493 (1999)

[9] E. Burgos, H.Ceva, Physica A284, 489 (2000)

[10] Within the rules for the BAM, an agent that loses credits is in the position to use the fact that her losses are due to the repeated action of not attending the bar. She could then use this to update her attendance strategy increasing that probability. This speeds up the convergence to the accepted value of $\mu$ in a dramatic way, but adds no conceptual change to the structure of the asymptotic behavior of the system. We did not use it in order to stress that the different behaviors are only due to the different use of the available information.

[11] A.Goldberg Genetic Algorithms in Search, Optimization, and Machine Learning, Addison - Wesley (1989).

[12] Note that $\mathcal{C}$ differs from $\sigma_{M}$ introduced previously, because the former is the dispersion at time $t$ with respect to the allowed attendance $N \mu$, while the later is the dispersion of the asymptotic attendance with respect to its average. 


\section{FIGURES}

FIG. 1. GMG and BAM without quenching. In this case, $N=1001, G=1$ and $\mu=600 / 1001$. The data is averaged over 200 histories. In (a) we show $P_{\infty}(p)$ for both cases; (b) illustrates the time evolution of the reduced attendance $M(t)$ and $C_{s}(t)$, the fraction of moving agents. Notice that $C_{s}(t)$ does not vanish

FIG. 2. BAM and GMG with quenching, for the same values of $N$ and $G$ than in the previous figure, but $\mu=800 / 1001$. In (a) we show that the shape of $P(p)$ has little changes for the BAM, while the GMG distribution is strongly modified. Correspondingly, in (b) one can see that GMG does not reach the allowed assistance $(M \neq \mu)$ because the system stops evolving $\left(C_{s}=0\right)$ very rapidly. Data for GMG is averaged over 3000 histories. In the broken x-axis we show that BAM does not quench even after $10^{6}$ time steps

FIG. 3. (a) Reduced attendance as a function of $\mu$, for $N=101$. It can be seen that $\mu_{c r}$ is a function of $G$. Size effects are shown in the inset. (b) transition from a non-quenched $\left(\varphi_{20} \neq 1\right)$ to a quenched state $\left(\varphi_{20}=1\right)$

FIG. 4. Phase diagram for the GMG in the $\mu-G$ plane. $(\triangle)$ shows an analytic solution for a $\delta$-type initial condition, while $(\square$ and $(\diamond)$ correspond to numerical solutions starting from a uniform distribution. The critical border for the BAM with $\delta$-type initial conditions would be a vertical line at $G \simeq 1,22$ for $N=100$

FIG. 5. Change of $P(p)$ with $G$, for $\mu=0.6$

FIG. 6. The roots $p_{p}$ and $p_{c}$ of Eqs.(9) and (10) are exemplified for $N=20$ and $n=14$. These values are chosen for the sake of clarity of the figures. Notice that as $G$ increases $p_{c}$ becomes smaller and $p_{p}$ becomes larger

FIG. 7. Quenching for the BAM for $\delta$-type initial conditions is possible for $G>G_{c r}$. The roots

$p_{p}$ and $p_{c}$ are ploted as a function of $G$ and the indicated values of $\mu$. Both roots are equal for $G=G_{c r}$. The value of $G_{c r}$ is largely independent of $\mu$.

FIG. 8. Distributions $P(p)$ for the GMG (right axis), obtained from three initial conditions $\delta\left(p-p_{0}\right)$. We also show $P_{w}$, (left axis) to help to understand the evolution of the systems. The roots $p_{<}$and $p_{>}$correspond to the two roots $P_{w}=1 / 2$. In this case, $\mu=75 / 101$

FIG. 9. (a) $G_{\text {crit }}$ vs $1 / N$ up to $N=1000$. Eq.14 provides an excellent fit that can not be distinguished from numerical data for GMG, $G=1, \mu=0.5$; (b) $p_{c r}$ as a function of $1 / N$ for the same values of $\mu$. 
FIG. 10. Annealing in the GMG 

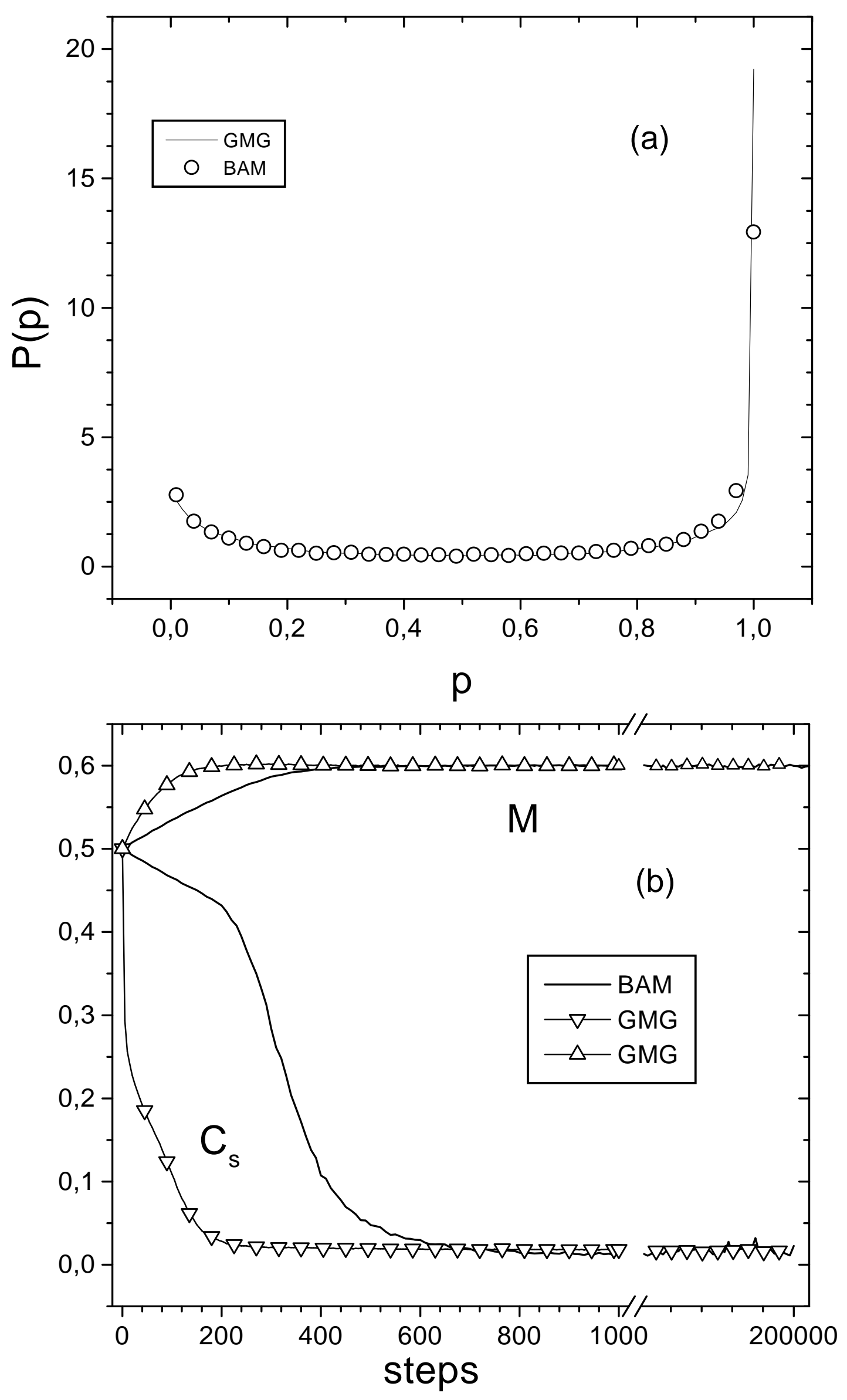



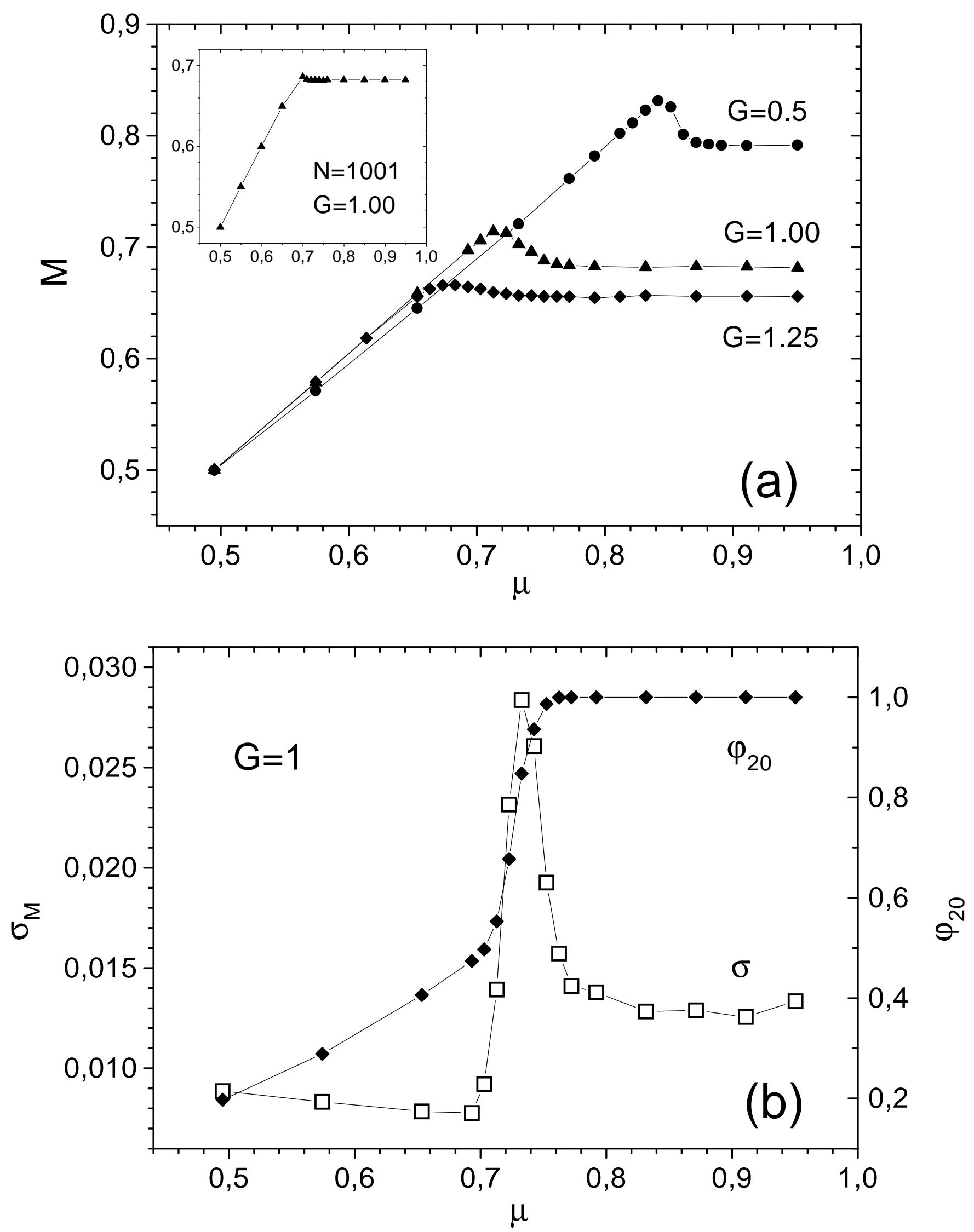


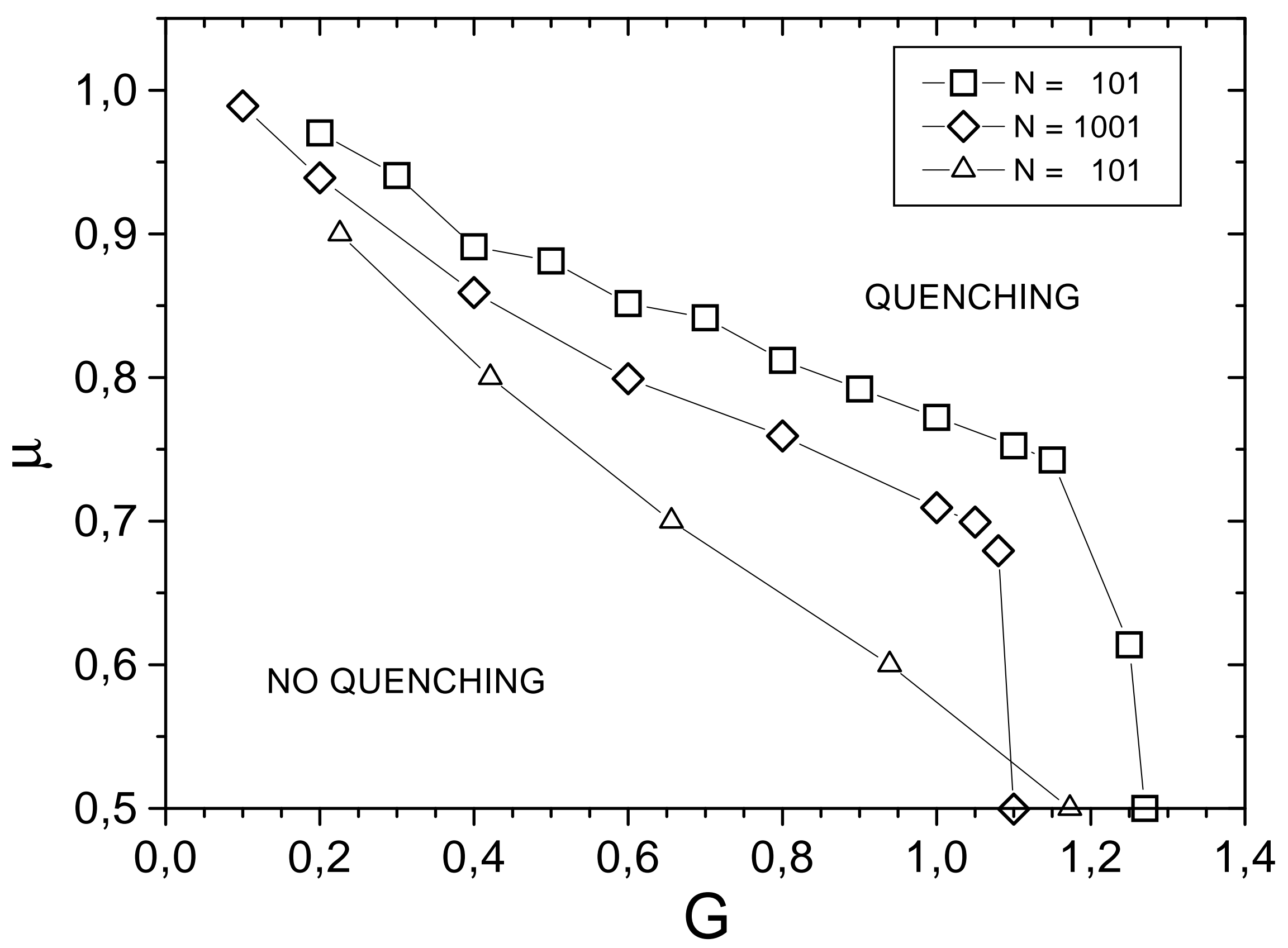




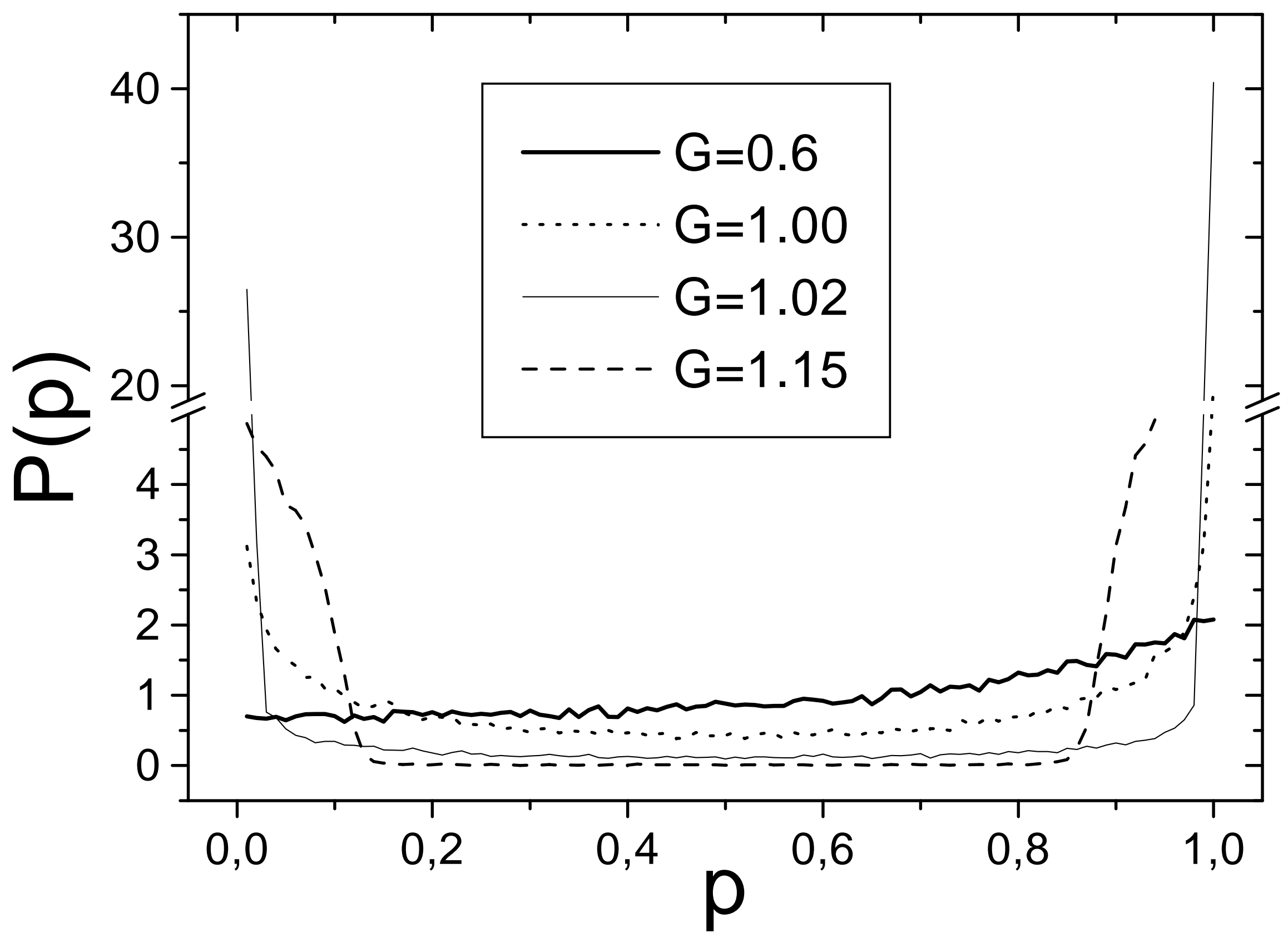









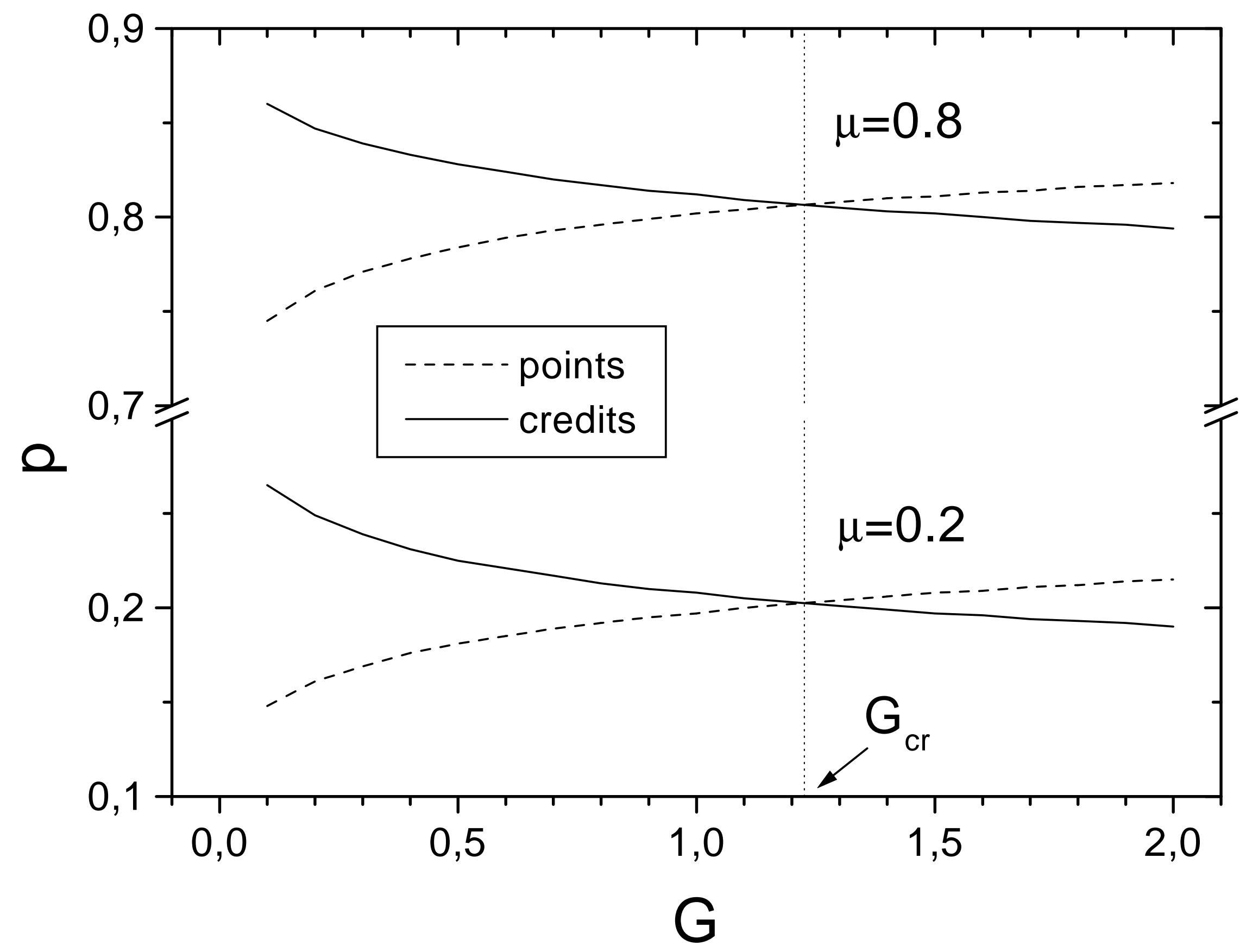




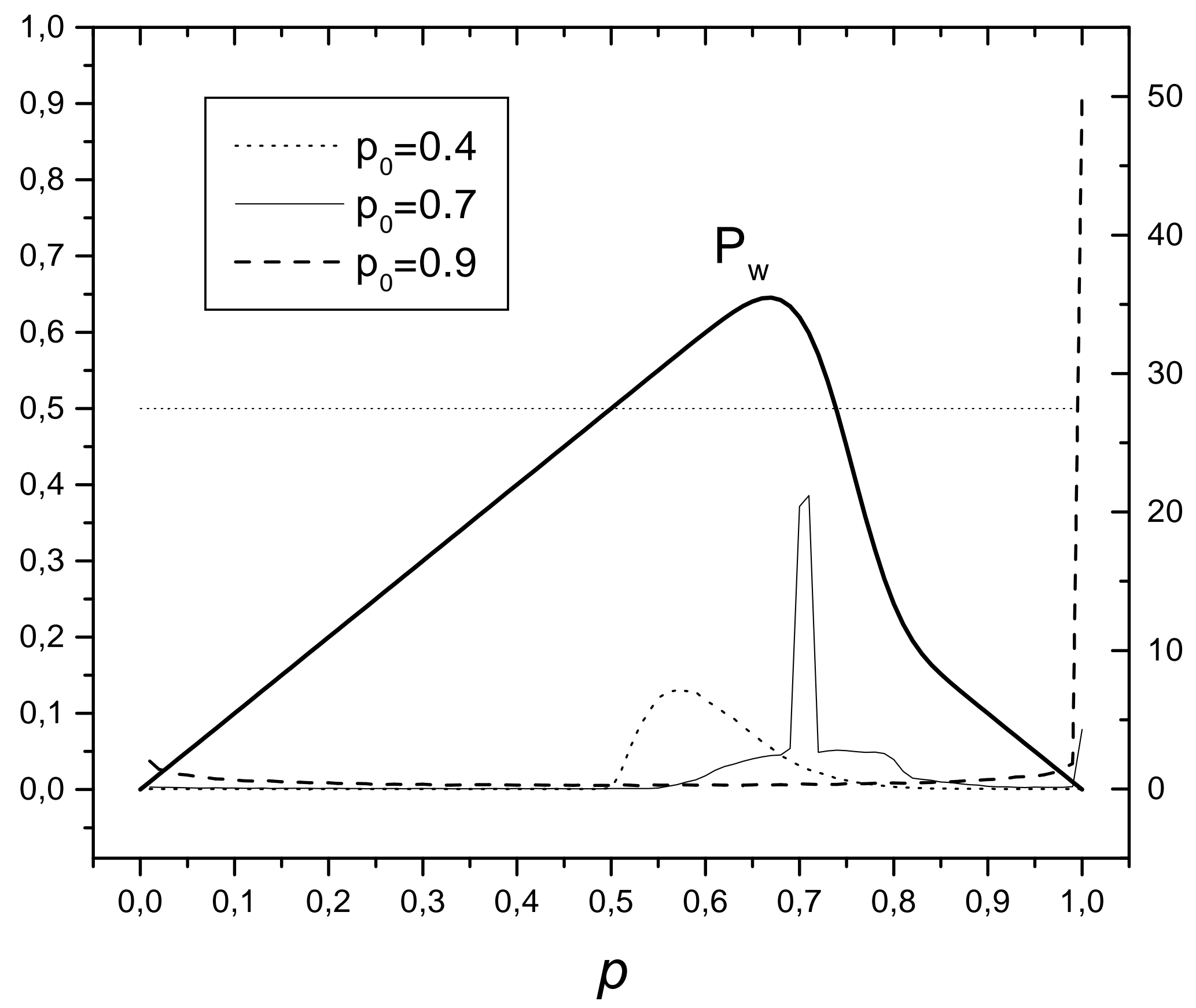



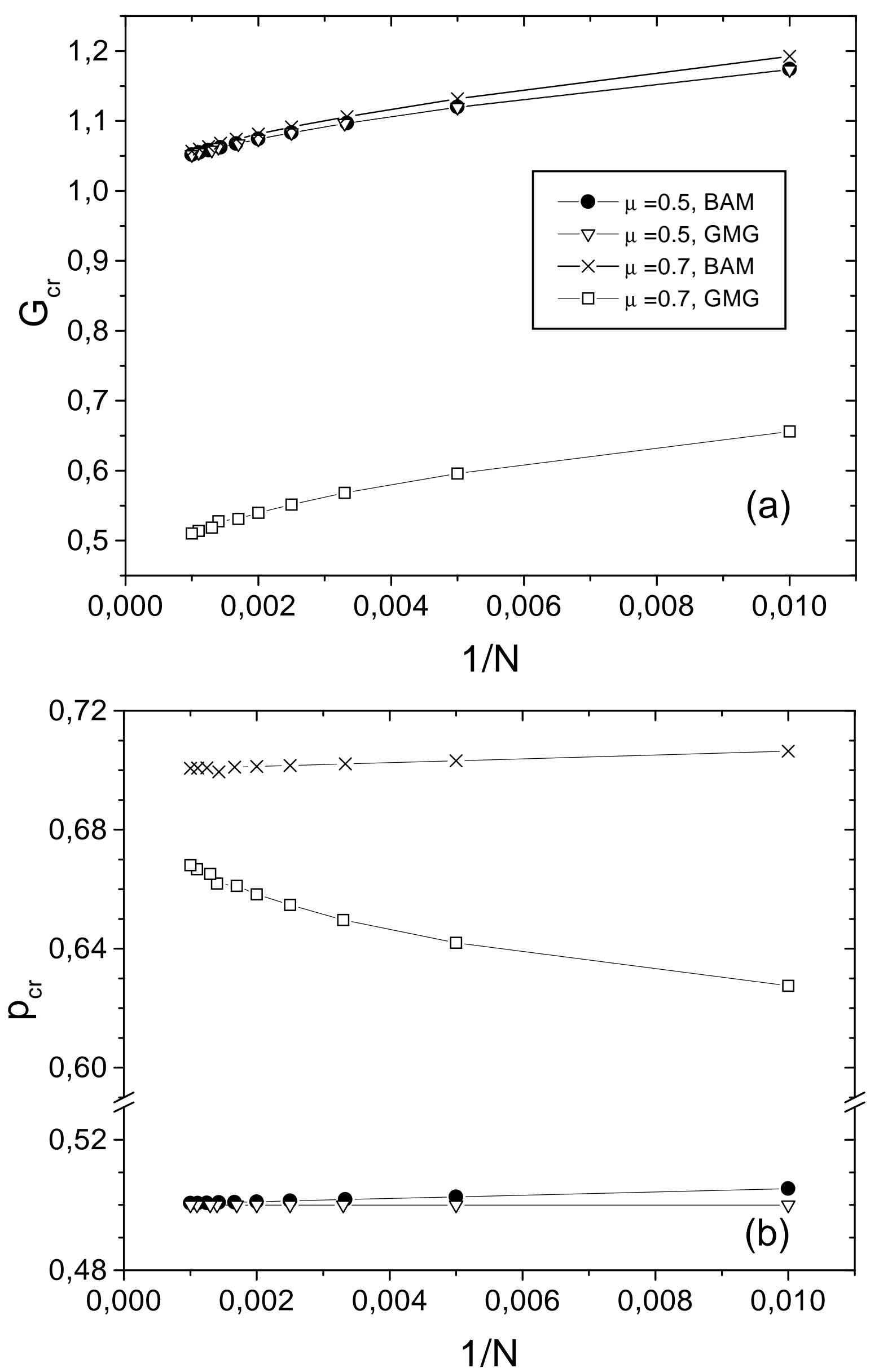

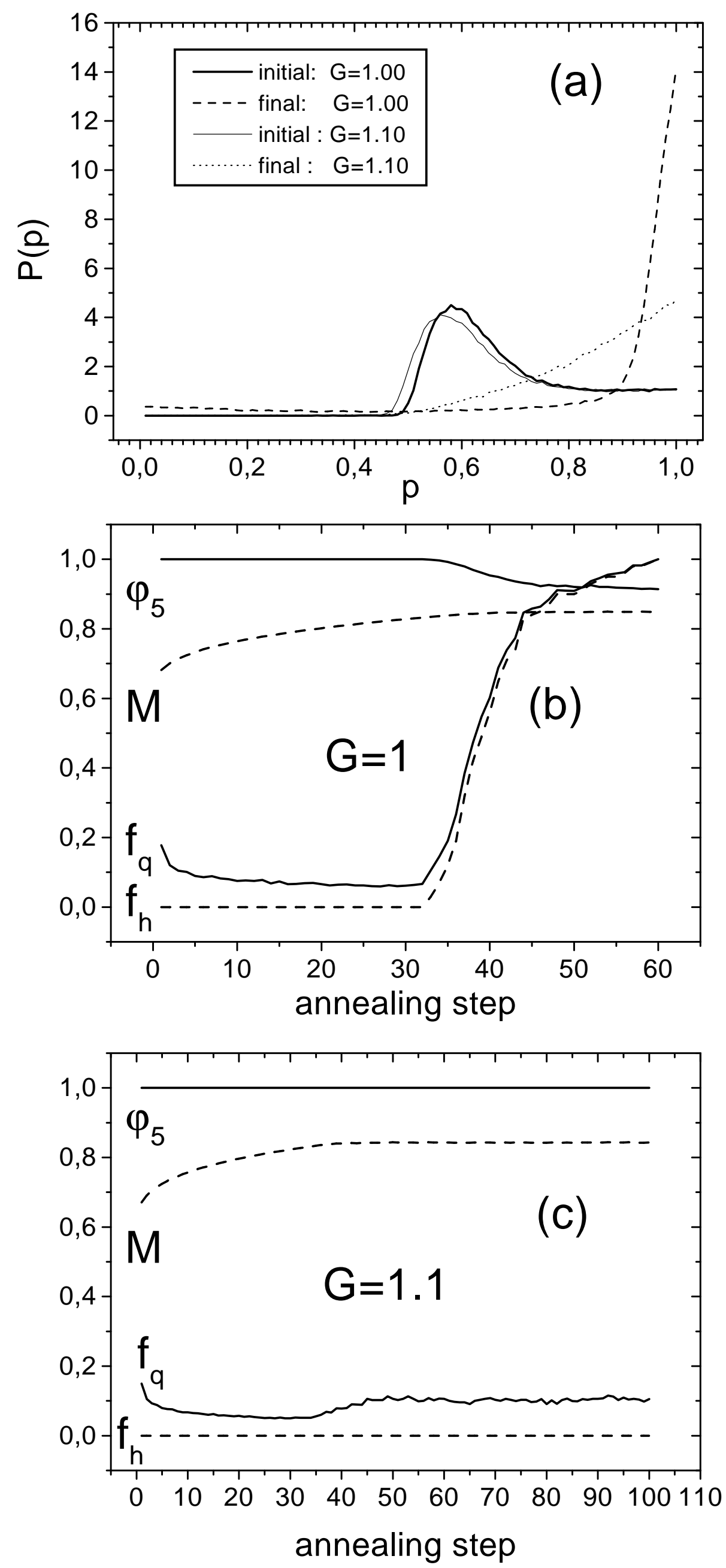